\title{
Space-Time Channel Model for Rain-Affected Communication Networks
}

\author{
Guangguang Yang, Boris Gremont ${ }^{1}$, Linda Yang, Mohammed Ibrahim and Li Bai
}

\begin{abstract}
This paper presents a new comprehensive spacetime model of rainfall rate and its associated rainfield synthesizer called GRAM (Grid Rain Attenuation Model). A great deal of care is placed on explicitly including the rain/no rain condition so that the planning and simulation of high frequency radio wave communication systems can be achieved with full consideration of rain intermittency effects. We also look in some details at the modification of first and second order statistical descriptions of the rain precipitation rate upon application of space and time integration to point rainfall rate.
\end{abstract}

Index Terms - rain, satellite, model, propagation, radio wave, simulation, stochastic.

\section{INTRODUCTION}

$\mathrm{R}$ AIN attenuation is a major impairment for wireless line of sight communications above $10 \mathrm{GHz}$ as often used for high frequency satellite links or microwave terrestrial links [1]. So the users' communication needs and weather condition are required to be taken into account in the design of next generation of satellite systems.

The experimental characterization of rain based on measurements (e.g. using rain gauges or rain radars) at different integration scales is extremely important to communication systems design [2] since as we shall show shortly, integration over different space and time scales changes the first and second order characteristics of rainfall rate and thus space and time averaging will change raininduced attenuation on radio links. The present paper therefore addresses the likely scenario that measurements of precipitation at spatial scales say $L$ and $T$ or alternatively first and second order statistical information about precipitation for scales $L$ and $T$ are available to the communication systems engineer but the realistic planning and design of systems

Guangguang Yang, University of Portsmouth, Anglesea Building, Anglesea Road, Portsmouth, PO1 3DJ, UK. Email: guangguang.yang@myport.ac.uk. Tel: +442392842587 .

${ }^{1}$ Dr Boris Gremont passed away on 20 December 2013.

Linda Yang, University of Portsmouth, Anglesea Building, Anglesea Road, Portsmouth, PO1 3DJ, UK. Email: linda.yang@port.ac.uk. Tel: $+442392842587$

Mohammed Ibrahim, University of Portsmouth, Anglesea Building, Anglesea Road, Portsmouth, PO1 3DJ, UK. Email: mohammed.ibrahim@myport.ac.uk. Tel: +442392842587 .

Li Bai, University of Portsmouth, Anglesea Building, Anglesea Road, Portsmouth, PO1 3DJ, UK. Email: up793863@myport.ac.uk. would require descriptions of rain at different usually smaller scales $L^{\prime}$ and $T^{\prime}$. For example, rain radar maps are typically produced every 5 or 15 mins. However, network planners and designers of physical layer fade mitigation techniques [3][4] would require knowledge of rain variations over much shorter time scales of the order of the second or even less.

Typically, rain plays a significant role in radio wave propagation and inherits high variability in both space and time [5]. This has caused intriguing challenges to satellite system planners and designers. Thus, better understanding of rain variations, together with the detailed knowledge of spacetime scaling of rainfield characteristics, particularly at the smaller scales, is of importance to radio wave transmissionoriented applications. Because of this the modeling of rainfield variability is of vital importance to rain-induced attenuation [6] as well as for the prediction of communication systems performance. Given that rain modeling at varying scales gives cause to substantial differences in impact on radio propagation, Valeria. Et al [7] examined the variability of rain in a wide range of time scales from $30 \mathrm{~min}$ to $720 \mathrm{mins}$. Luini and Capsoni in [8] investigated the space- and time- averaging of rainfall rate to obtain the spatial correlation with different scale dependencies. In particular, our previous work [9] developed a set of equations that enabled the prediction of space-time estimates of so-called "key characteristics" with a high level of accuracy throughout the whole range of distances and/or time lags. All these can be used by the rainfield simulator to yield attenuation fields spanning a range of space and time scales. An interesting model is proposed by Bell [10] based on some constraints, i.e. rainfield is spatially homogeneous and isotropic. As a result, rain rate can be reasonably generated from a portion of a Gaussian field $g(\mathbf{x}, t)$ that exceeds a threshold. Then later in [11], Kundu and Bell developed a statistical model that can study the correlation functions of rain in space and time simultaneously. In consequence they introduced a stochastic fractional diffusion model and studied the scaling behavior of both areaaveraged and time-averaged rain statistics [12]. They found that the second moment rainfields statistics exhibit invariance under the combined rescaling of the space-time variables. In 2011, Kundu and Siddani [13] explored the dependence of probability of zero-rain on the area- and/or time-averaged scales variations and proposed an exponential formula to fit the measurements and which provides excellent approximation. A particular example is our recent development [14] (as extension of [9]) that focused on the 
space-time averaging on the dynamic-statistical properties of rainfields. It shows that scaling of rain has great impact on rainfields simulation and more remarkably, the proposed statistical model and interpolation approach can provide reasonable estimations of rain properties at high space-time resolutions up to $10 \mathrm{~s}$ in time domain and $50 \mathrm{~m}$ in space domain. Similar work has been done by other researchers, but they only focused on a specific rain property, i.e. the spatial correlation function of rain (see [8]) or the probability of rain occurrence (see [15]). The ever-growing demand for characterizations of rainfield at multi-resolutions, particularly at smaller scales, is a major requirement of communication networks. This paper introduces a new space-time model that investigates how the rainfield, as well as its relating properties, change with varying integration lengths in space and time domains.

The rest of this paper is organized as follows. In section II, we introduce a space-time model for point rainfall rate. We also describe in details the space-time specifications and field synthesis. In section III, we investigate the scaling in the space and time domain for interpolation of the rainfields together with the description relating to the fractional rainy area. A set of equations useful for the prediction of rain attenuation for satellite and terrestrial links are provided in Section IV. Conclusions are drawn in Section V.

\section{SPACE-TIME MODEL OF POINT RAINFALL RATE}

\section{A. Statistical model description}

We are interested in the rainfall rate $R(\mathbf{x}, t)$ at point where $\mathbf{x}=\left[\begin{array}{ll}x_{1} & x_{2}\end{array}\right]$ represents the two spatial coordinates on the horizontal plane and $t$ represents the time variable. On the long run, the statistic of rainfall rate is assumed to be conditionally lognormal [1][16], i.e. it has a mixed density function:

$$
f(R)= \begin{cases}1-f & \text { for } R=0 \\ \frac{f}{\sqrt{2 \pi} \sigma R} \exp \left(-\frac{1}{2}\left(\frac{\ln R-m}{\sigma}\right)^{2}\right) & \text { for } \mathrm{R}>0\end{cases}
$$

where $f$ denotes the probability of occurrence of rain at point $\mathbf{x}$ and $(m, \sigma)$ are the two parameters required for specifying the statistics of rain when $R>0$. We also remark that $f$ is also the long run probability of rain on a rain map with total area $\mathcal{A}$ i.e. $f=\mathcal{A}_{\text {rainy }} / \mathcal{A}$, here $\mathcal{A}_{\text {rainy }}$ stands for the rainy area within the studied area. It is straightforward to find that the first and second order moments of rainfall rate are:

$$
E\{R\}=f \exp \left(m+\frac{\sigma^{2}}{2}\right) \equiv \mu_{R}
$$

and

$$
E\left\{R^{2}\right\}=f \exp \left(2 m+\sigma^{2}\right),
$$

and the variance $\sigma_{R}^{2} \triangleq E\left\{(R-E\{R\})^{2}\right\}$ of $R$ is:

$$
\sigma_{R}^{2}=f\left[\exp \left(\sigma^{2}\right)-1\right] \exp \left(2 m+\sigma^{2}\right)
$$

Let us consider a Gaussian field $g(\mathbf{x}, t)$ with zero mean and unit variance. On the long run, over many Gaussian 2D maps $g_{t}(\mathbf{x})$, the non-linear transformation:

$$
\mathcal{T}\left\{g_{t}(\mathbf{x})\right\}=\left\{\begin{array}{cl}
0, & g_{t}(\mathbf{x})<g_{0} \\
\exp \left(\sigma Q^{-1}\left(\frac{Q\left(g_{t}(\mathbf{x})\right)}{f}\right)+m\right), & \text { otherwise }
\end{array}\right.
$$

will produce a rain field with the pdf in Eq. (1) provided we choose the threshold $g_{0}$ so that:

$$
f=\int_{g_{0}}^{\infty} \frac{1}{\sqrt{2 \pi}} \exp \left(-\frac{u^{2}}{2}\right) d u \equiv Q\left(g_{0}\right)
$$

i.e. by choosing $g_{0}=Q^{-1}(f)$ where we introduce the functional inverse of the complementary error function $Q(\mathrm{x})$.

It is important to note that transformation (5) allows to link the correlation coefficient of the Gaussian field $\rho$ with that of the rain field denoted by $\rho_{R}$. In particular, for two Gaussian zero mean unit variance random variables $g$ and $h$, the correlation function of rainfall rate is obtained through a double integral [17]:

$$
E\{R(u) R(v)\}=\int_{g_{0}}^{\infty} \int_{g_{0}}^{\infty \infty} \mathcal{T}\{u\} \mathcal{T}\{v\} f(u, v ; \rho) d u d v
$$

which we concisely write:

$$
E\{R(g) R(h)\} \triangleq \Xi(\rho),
$$

and the joint pdf of the Gaussian field takes the form (see [17])

$$
f(u, v ; \rho)=\frac{1}{2 \pi \sqrt{1-\rho^{2}}} \exp \left(-\frac{1}{2} \cdot \frac{\left(u^{2}-2 \rho u v+v^{2}\right)}{1-\rho^{2}}\right)
$$

and obviously $|\rho| \leq 1$. The correlation coefficient $\rho_{R}$ of point rainfall rate is thus computed as (note that the numerator is the covariance function $c_{R}$ ):

$$
\begin{aligned}
\rho_{R} & =\frac{E\{R(u) R(v)\}-(E\{R\})^{2}}{E\left\{(R-E\{R\})^{2}\right\}} \\
& =\frac{\Xi(\rho)-f^{2} \exp \left(4 m+4 \sigma^{2}\right)}{f\left[\exp \left(\sigma^{2}\right)-1\right] \exp \left(2 m+\sigma^{2}\right)} \\
& =\Upsilon(\rho)
\end{aligned}
$$

If alternatively we know the correlation of rainfall rate, we can obtain the correlation of the Gaussian field using the functional inverse $\rho=\Upsilon^{-1}\left(\rho_{R}\right)$. We note that Eq. (9) can be rearranged to $E\{R(u) R(v)\}=\rho_{R} \sigma_{R}^{2}+u_{R}^{2}$.

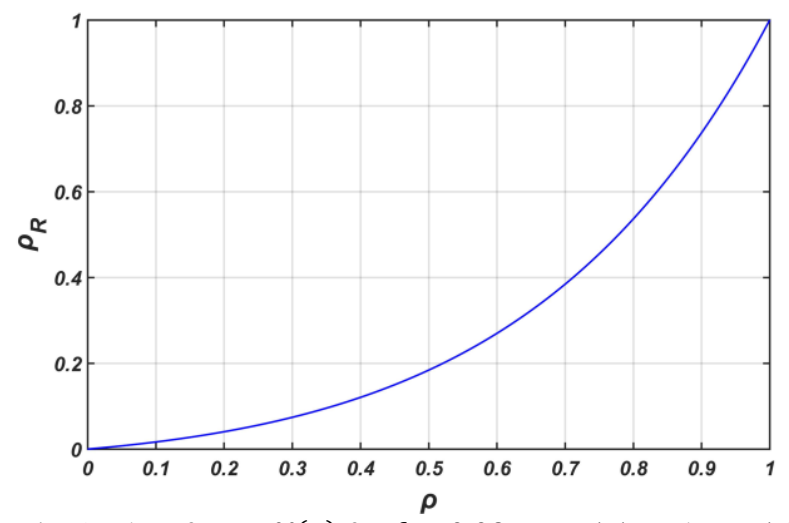

Fig. 1: Plot of $\rho_{R}=\Upsilon(\rho)$ for $f=0.09, \mathrm{~m}=1.1$, and $\sigma=1.3$. 
A typical example of the computation of $\Upsilon(\rho)$ is shown in Fig. (1) for particular values of the main statistical parameters $(f, m, \sigma)$ of rainfall rate. We also note that $\Upsilon(\rho)$ is very well approximated by a fifth order polynomial:

$$
\Upsilon(\rho) \approx \sum_{k=0}^{5} p_{k} \rho^{5-k}
$$

which is important to numerically convert the Gaussian correlation function to that of rainfall rate (In our particular example the polynomial coefficients are $p_{0}=0.2584, p_{1}=$ $-0.0 .0735, p_{2}=0.5158, p_{3}=0.1940, p_{4}=0.1084$, $\left.p_{5}=-0.0002\right)$. As we will shortly see, we use this polynomial conversion extensively to describe and validate the model/synthesizer described below. Obviously, this polynomial fit must be recomputed for each set of model statistical input parameters $(f, m, \sigma)$.

\section{B. Space-time specifications and field synthesis}

In this paper, we consider a space-time separable space-time model of rainfall rate, for example the model proposed by Bell [10]. In particular, we assume that the time variations of the Gaussian field $g(\mathbf{x}, t)=g_{t}(\mathbf{x})$ (the independent variable is $t$ and that $g(\mathbf{x})$ is a 2D Gaussian field a spatial correlation function to be described later) is time differentiable and that it satisfies the double stochastic PDE (Partial Differential Equation):

$$
\frac{\partial g_{t}}{\partial t}+\beta(\mathbf{x}) g_{t}(\mathbf{x})=F_{t}(\mathbf{x})
$$

where the forcing function $F(\mathbf{x}, t)$ is an Ornstein-Uhlenbeck process driven by a Wiener process $d W(\mathbf{x}, t)$ described by the Ito SDE (Stochastic Differential Equation):

$$
d F_{t}(\mathbf{x})=-\frac{1}{\tau_{c}} F_{t}(\mathbf{x}) d t+\sigma_{w} d W_{t}(\mathbf{x})
$$

The model input parameters $\beta(\mathbf{x})$ is a location-dependent quantitative descriptor for the temporal characteristics of rainfields. We note that the model allows for a different value of $\beta$ at each location. Time constant $\tau_{c}$ is chosen to be small compared to the expected smallest time scales associated with precipitation processes. The standard deviation $\sigma_{w}$ of the Wiener process is chosen so that the variance of $g_{t}(\mathbf{x})$ is unity. Numerically, the exact stepping formula for $F_{t}(\mathbf{x})$ can be derived from solving and then discretizing Eq. (12):

$$
F_{t+\Delta t}(\mathbf{x})=F_{t}(\mathbf{x}) \cdot e^{-\frac{\Delta t}{\tau_{c}}}+\sigma_{w} \mathcal{N}_{t}(\mathbf{x}) \sqrt{\frac{1-e^{-\frac{2 \Delta t}{\tau_{c}}}}{2 \tau_{c}}}
$$

where $\mathcal{N}_{t}(\mathbf{x})$ is a time-domain uncorrelated Gaussian zero mean unit variance random matrix and $\Delta t$ is the simulation time-step resolution. This, with the discretized version of Eq. (11) forms the core of the temporal synthesis for the spacetime rainfiled simulator.

It is straightforward to demonstrate that the steady-state temporal spectral density function $S_{g}(f, \mathbf{x})$ of the Gaussian field at location $\mathbf{x}$ is of second-order and is given by:

$$
S_{g}(f, \mathbf{x})=\frac{\sigma_{w}}{4 \pi^{2} f^{2}+\beta(\mathbf{x})^{2}} \times \frac{\left(\frac{1}{\tau_{c}}\right)^{2}}{4 \pi^{2} f^{2}+\left(\frac{1}{\tau_{c}}\right)^{2}}
$$

The time (auto-) covariance function of the Gaussian field denoted $\quad c_{g}(\tau) \triangleq E\left[\left(g(\mathbf{x}, t)-m_{g}(\mathbf{x}, t)\right)\left(g\left(\mathbf{x}^{\prime}, t^{\prime}\right)-m_{g}\left(\mathbf{x}^{\prime}, t^{\prime}\right)\right)\right]$ with $\tau=\left|t-t^{\prime}\right|$ can be computed using the inverse Fourier transform:

$$
c_{g}(\tau)=\int_{-\infty}^{\infty} e^{i 2 \pi f \tau} \mathcal{S}_{g}(f) d f
$$

yielding:

$$
c_{g}(\tau)=\frac{\sigma_{w}}{2 \beta} \times \frac{e^{-\beta|\tau|}-\beta \tau_{c} e^{-\frac{|\tau|}{\tau_{c}}}}{1-\beta^{2} \tau_{c}^{2}}
$$

We need to have unit variance for zero time lags so $\sigma_{w}=2 \beta\left(1+\beta \tau_{c}\right)$. Using Eq. (10), we can transform the correlation in Eq. (16) to get the time autocorrelation of rainfall rate. This is displayed in Fig. (2). We have also plotted the best fit exponential to the temporal correlation of rainfall which is clearly very good. This indicates that, to a very good approximation, the temporal correlation of rainfall rate can be assumed to have a second order spectral density function. We also remark that the rain correlation function is much more accurate than that of the Gaussian variable.

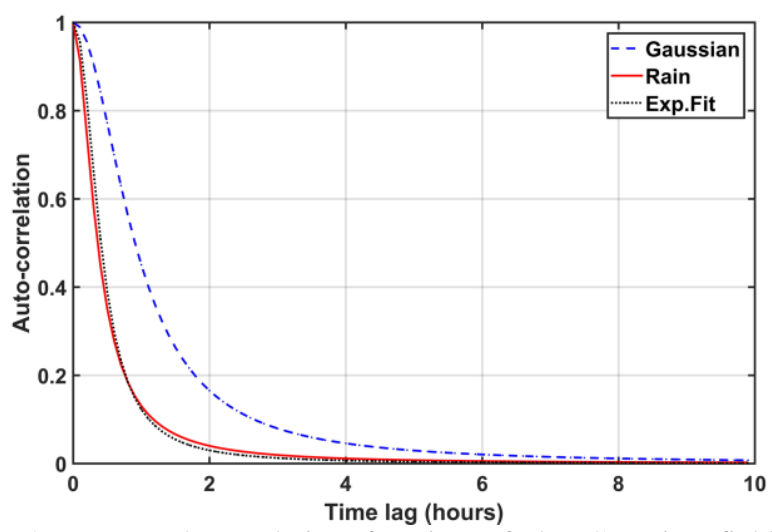

Fig. 2: Temporal correlation function of the Gaussian field, the rainfall rate and its associated exponential best fit with $\beta \approx 5.2 \times$ $10^{-4} \mathrm{~s}$.

In order to complete the model, we now need to describe the spatial spectrum of the precipitation field. In GRAM, all the field in Eq. (11) and Eq. (12) have the same spatial spectrum $\mathcal{S}(k)$ where we assume that the rain field is isotropic space homogeneous and thus in $\mathbb{R}^{2}$ its correlation function $c_{g}(\tau)$ can be obtained from the spatial spectral density function using [18]:

$$
c_{g}(r)=2 \pi \int_{0}^{\infty} k S(k) J_{0}(k r) d k
$$

where $k=\sqrt{{k_{1}{ }^{2}+k_{2}{ }^{2}}^{2}}$ denotes the angular spatial frequency. The radial spatial spectral density function is chosen to be:

$$
S_{g}(k)=\tilde{a}^{2}(\tilde{a} k)^{\tilde{n}-1} K_{\tilde{n}-1}(\tilde{a} k)
$$

where $K_{\alpha}(x)$ denotes the modified Bessel function of the second kind. Thus, for $r>0$ and $\tilde{n}>-\frac{3}{4}$ : 


$$
c_{g}(r)=\left(\frac{\tilde{a}^{2}}{\tilde{a}^{2}+r^{2}}\right)^{\tilde{n}}
$$

Using Eq. (10) we can easily obtain the radial spatial correction function $\rho_{R}(r)$ of rainfall rate itself. This is plotted in Fig. (3) where we show the theoretical correlation function of rainfall rate $c_{R}(r)=\Upsilon\left(c_{g}(r)\right)$ (solid line) obtained from Eq. (19) assuming the parameters $\tilde{a}^{2}=1.44$ and $\tilde{n}=0.37$.

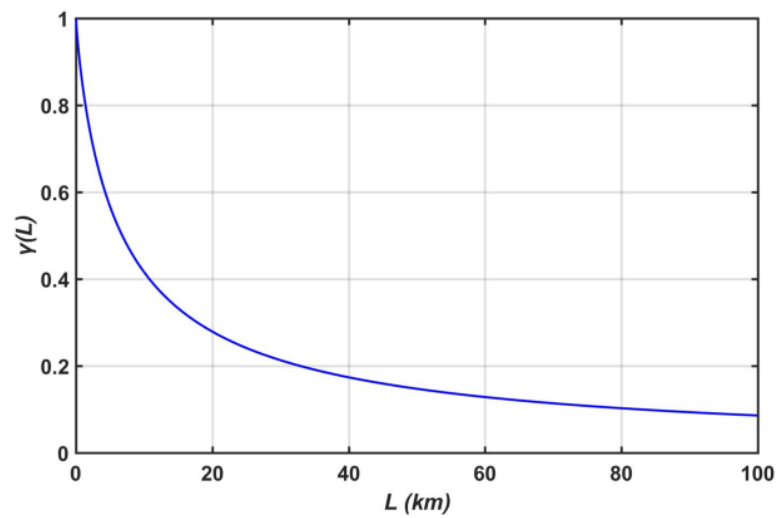

Fig. 3: Spatial correlation function $c_{R}(r)$ of rainfall rate derived from the Gaussian correlation given in Eq. (19) with $\tilde{a}^{2}=1.44$ and $\tilde{n}=0.37$.

We also compare this theoretical spatial correlation function with the empirical radial correlation function $c_{R}^{\text {exp }}(r)$ of rainfall rate measured from rain radar maps obtained by the UK NIMROD network which takes the form:

$$
c_{R}^{e x p}(r)=\frac{\tilde{b}}{\tilde{b}+|r|^{\tilde{m}}}
$$

where good experimental fit was obtained for $\tilde{b}=10.0$ and $\widetilde{m}=0.21$ in the region in the vicinity of Portsmouth (UK) [9].

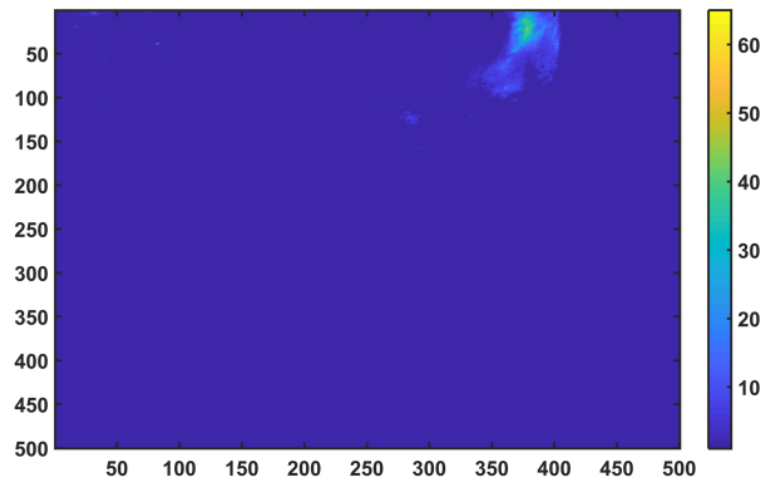

Fig. 4: Simulated spatial rainfall rate field in $m m / h$ with $f=0.09$, $m=1.4, \sigma=1.1$ obtained upon application of the turning band algorithm, here the pixel resolution is $1 \mathrm{~km}$.

The comparison shows excellent match over the whole range of distances. In order to produce space-time fields, the GRAM simulator must first produce an initial rainfield map with the required spatial correlation function $c_{g}(r)$. There are many ways of achieving this and we present one algorithm called the Tuning Band Method in the Appendix. A typical rainfield map $g_{t}(\mathbf{x})$ spanning a $500 \mathrm{~km} \times 500 \mathrm{~km}$ area is displayed in Fig. 4. The probability of rain occurrence was set to $39 \%$. One can see quite a deep rain event in the top right corner with a peak rainfall rate of the order of $40 \mathrm{~mm} / \mathrm{h}$ with a characteristic size of about $60 \mathrm{~km} \times 40 \mathrm{~km}$.

\section{INTEGRATED RAINFALL RATE FIELDS}

\section{A. Space and time averaging}

In practice, the rainfield space-time data produced by for example rain radar systems is averaged over a duration $T$ and over an area of size $L \times L$. We should therefore look into the impact of integration time and integration length on the first and second order statistical characteristics of precipitation rain fields. This is particularly useful in order to re-scale in space or time exponentially gathered data that has the wrong space or time resolution for the particular study of interest. Time interpolation or space interpolation (otherwise known as down-scaling) of rainfall rate fields can be seen as inverse operations.

Let us first consider the time averaging over a duration $T$ (we can call this temporal resolution) of the precipitation rate over successive time $T$ - wide intervals located at $k T$ with $k \in \mathbb{N}$ defined by:

$$
R_{T}(\mathbf{x}, t) \triangleq \frac{1}{T} \int_{t-\frac{T}{2}}^{t+\frac{T}{2}} R\left(\mathbf{x}, t_{u}\right) d t_{u}
$$

Since we assume that the space-time rainfall rate process is time-homogenous, it is straight forward to verify that $E\left[R_{T}(k T)\right]=E[R(x, t)]=\mu_{R}$ which thus on the long-term equals Eq. (2). The variance $\operatorname{Var}\left[R_{T}(\mathbf{x}, k T)\right] \triangleq \sigma_{R}^{2}(T)$ of the time-averaged precipitation rate is more interesting since (using Eq. (9) and [17]):

$$
\begin{aligned}
\sigma_{R}^{2}(T) & =\frac{\sigma_{R}^{2}}{T^{2}} \int_{0}^{T} \int_{0}^{T} c_{R}(|u-v|) d u d v \\
& =\frac{\sigma_{R}^{2}}{T^{2}} \int_{0}^{T} \int_{0}^{T} \Upsilon(|u-v|) d u d v \\
& =\frac{2 \sigma_{R}^{2}}{T} \int_{0}^{T}\left(1-\frac{\tau}{T}\right) \Upsilon\left(c_{g}(\tau)\right) d \tau
\end{aligned}
$$

where $\sigma_{R}^{2}$ is the variance of point rainfall rate as given in Eq. (4) and the temporal correlation function of the Gaussian field is given in Eq. (16). This allows us to introduce the variance reduction function $\gamma(T) \equiv \frac{\sigma_{R}^{2}(T)}{\sigma_{R}^{2}}$ :

$$
\gamma(T)=\frac{2}{T} \int_{0}^{T}\left(1-\frac{\tau}{T}\right) \Upsilon\left(c_{g}(\tau)\right) d \tau
$$

that describes how the point variance of rainfall rate is affected by the integration time. Function $\gamma(T)$ is plotted in Fig. (5) and shows that integrating the precipitation over a period of 4 hours would reduce the point variance by a factor of about 5 . The variance reduction function is particularly useful to rescale the variance. For example, if rainfall rate was measured by a system with integration time $T^{\prime}$ and we would like to estimate the variance we would get for another integration time $T$ then based on our definitions we can use the 
transformation formula $\frac{\sigma_{R}^{2}(T)}{\sigma_{R}^{2}\left(T^{\prime}\right)}=\frac{\gamma(T)}{\gamma\left(T^{\prime}\right)}$.

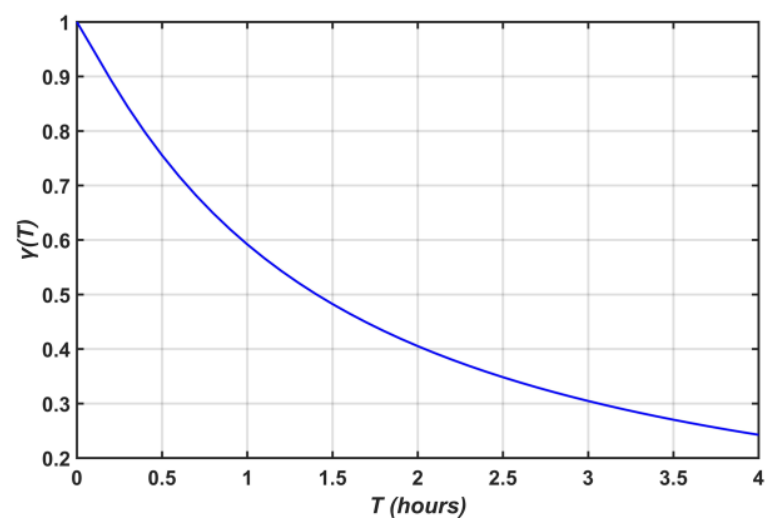

Fig. 5: Variance scaling function $\gamma(T)$ showing the reduction factor of the rainfall rate variance as a function of the integration time $T$.

It is also quite straightforward to generalize slightly Eq. (22) to get the temporal correlation function of the time-integrated rainfall rate:

$$
C_{R}^{T}(\tau)=\frac{1}{T^{2} \gamma(T)} \int_{0}^{T} \int_{0}^{T+\tau} \Upsilon\left(c_{R}(|u-v|)\right) d u d v
$$

This has been computed for different integration times from $T=1 \mathrm{~s}$ to $T=8 \mathrm{~h}$ and is displayed in Fig. (6). Our particular results indicate that the time correlation function is invariant and thus distinguishable for $T=1 \mathrm{~s}$ to $T=60 \mathrm{~s}$. However, for larger integration times there starts to be more and more significant differences indicating that the dynamic characteristics of rainfall rate time-series will become much slower with long range dependence.

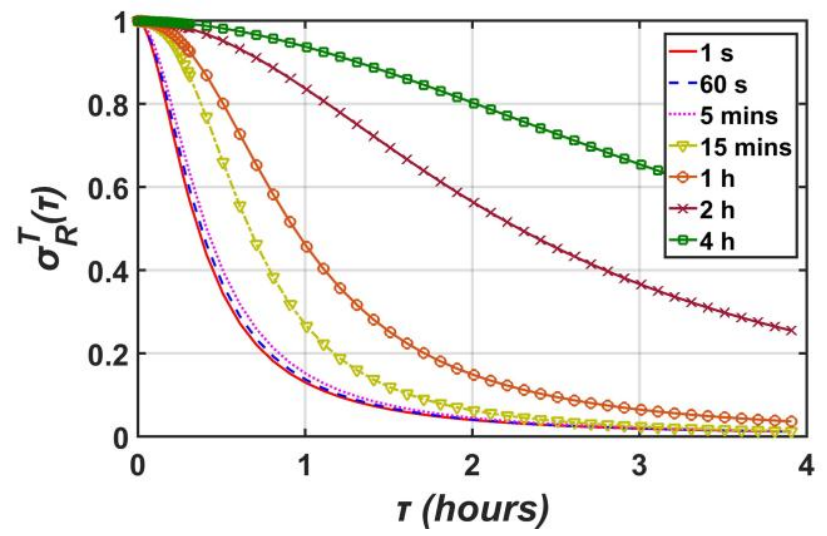

Fig. 6: Time correlation function of integral rainfall rate for different integration times from $T=1 s$ to $T=4 h$.

A similar analysis can be applied for the space integrated rainfall over a square of size $L \times L$ (i.e. $L$ denotes the space resolution) described by the double integral:

$$
R_{L}(\mathbf{x}, t) \triangleq \frac{1}{L^{2}} \int_{x_{1}-\frac{L}{2}}^{x_{1}+\frac{L}{2}} \int_{x_{2}-\frac{L}{2}}^{x_{2}+\frac{L}{2}} R\left(u_{1}, u_{2}, t\right) d u_{1} d u_{2}
$$

with $\mathbf{x}=\left(x_{1}, x_{2}\right) \in \mathbb{R}^{2}$. While space integration does not impact on the mean rainfall rate, the variance $\operatorname{Var}\left[R_{L}(\mathbf{x}, t)\right]$ of the integrated rainfield is modified by the integration process over a square area of $L^{2}$ and is given by $\sigma_{R}^{2}(L)=\sigma_{R}^{2} \Upsilon(L)$. The spatial variance reduction function is:

$$
\Upsilon(L)=\frac{1}{L^{2}} \int_{0}^{L} \int_{0}^{L} \Upsilon\left(c_{g}\left(\left|u_{1}-u_{2}\right|\right)\right) d u_{1} d u_{2}
$$

where this time we use the isotropic spatial correlation function given in Eq. (19). We display $\Upsilon(L)$ in Fig. (7). Like for the time integration case, the spatial variance reduction function can help the experimenter rescale the rainfall rate variance. In particular, if one knows the rain variance $=$ $\sigma_{R}\left(L^{\prime}\right)$ at scale $L^{\prime}$, the variance at scale $L$ is obtained using $\frac{\sigma_{R}^{2}(L)}{\sigma_{R}^{2}\left(L^{\prime}\right)}=\frac{\gamma(L)}{\gamma\left(L^{\prime}\right)}$.

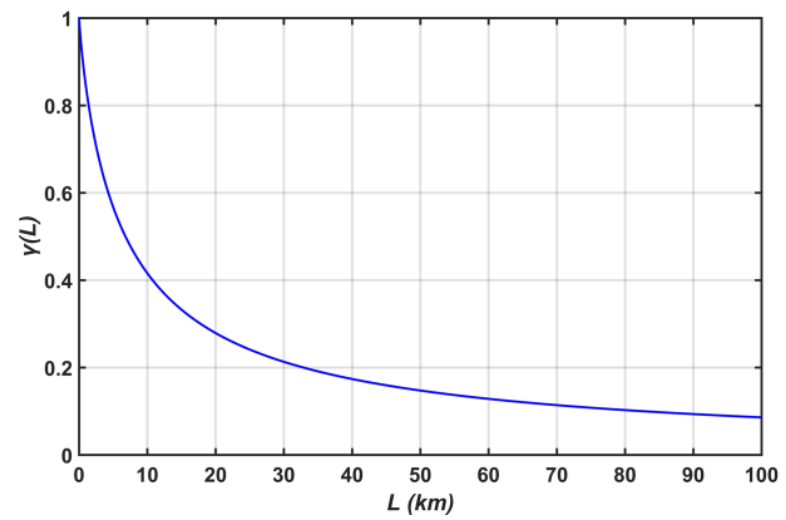

Fig. 7: Variance scaling function $\gamma(L)$ showing the reduction factor of the rainfall rate variance as a function of the spatial resolution $L$.

Since by assumption, the GRAM model is space-time separable, the space-time variance $\sigma_{R}^{2}(L, T)$ of rainfall rate, which gives the rainfall rate variance at arbitrary space and time resolutions is defined by:

$$
\sigma_{R}^{2}(L, T) \triangleq \sigma_{R}^{2} \Upsilon(L) \Upsilon(T)
$$

from which we introduce the space-time variance scaling function:

$$
\frac{\sigma_{R}^{2}(L, T)}{\sigma_{R}^{2}\left(L^{\prime}, T^{\prime}\right)}=\frac{\gamma(L) \gamma(T)}{\gamma\left(L^{\prime}\right) \gamma\left(T^{\prime}\right)}
$$

This general transformation will prove extremely useful for converting meteorological statistical databases of rainfall variances obtained at a (presumably) large space-time resolution to a smaller resolution, more in line with the planning requirements of communication systems (e.g. down to scales of the order of a few tens of meters and time intervals of the order of the second). After suitable changes of variable (see [17]), it can be shown that the spatial correlation function takes the form:

$$
\begin{gathered}
c_{R}^{L}\left(\epsilon_{1}, \epsilon_{2}\right)=\frac{2}{L^{2}} \int_{-L-\epsilon_{1}}^{L-\epsilon_{1}} \int_{-L-\epsilon_{2}}^{L-\epsilon_{2}}\left(1-\frac{\left|u+\epsilon_{1}\right|}{L}\right) \times \ldots \\
\ldots\left(1-\frac{\left|u+\epsilon_{1}\right|}{L}\right) c_{R}\left(\sqrt{u^{2}+v^{2}}\right) d u d v
\end{gathered}
$$

It is clear that this is symmetrical and thus $c_{R}^{L}\left(\epsilon_{1}, 0\right)=$ $c_{R}^{L}\left(0, \epsilon_{2}\right) \triangleq c_{R}^{L}(\epsilon)$ where $\epsilon$ denotes a spatial displacement. This is plotted in Fig. (8) for different spatial resolution $L$. 


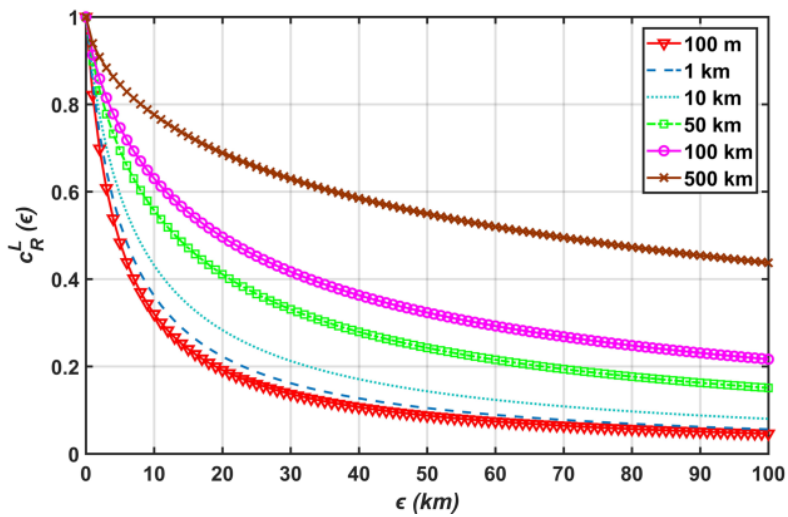

Fig. 8: Spatial correlation function of spatially-integrated rainfall rate for different spatial scales $L$ between $100 \mathrm{~m}$ and $500 \mathrm{~km}$.

In order to conclude this section, we now look at the impact of space and time averaging on rain intermittence. Rain occurrence is a very sporadic process in both space and time. However, in the ontext of the stochastic field model presented in this paper, rain intermittency is mostly covered through the rain occurrence probability $f$ that defines the mixed distribution given in Eq. (1). It seems quite difficult to propose a physical model for rain intermittence. Instead, it is more expeditious to recourse to experimental results as a basis to a phenomenological description of rain intermittence. In [9] the authors studies gridded rain radar maps from the UK NIMROD rain radar networks to produce the following experimental function for rain spatial intermittence (based on data for the whole year 2008 with integration time of $T=$ $5 \mathrm{~min})$ :

$$
f(L, T=5 \mathrm{~min})=1-(1-f) e^{-e L^{g}}
$$

where for the southern UK, the empirical constants are: $f=0.09, e=0.042$ and $g=0.85$ and $L$ is in $\mathrm{km}$. The goodness of fit is demonstrated in Fig. (9).

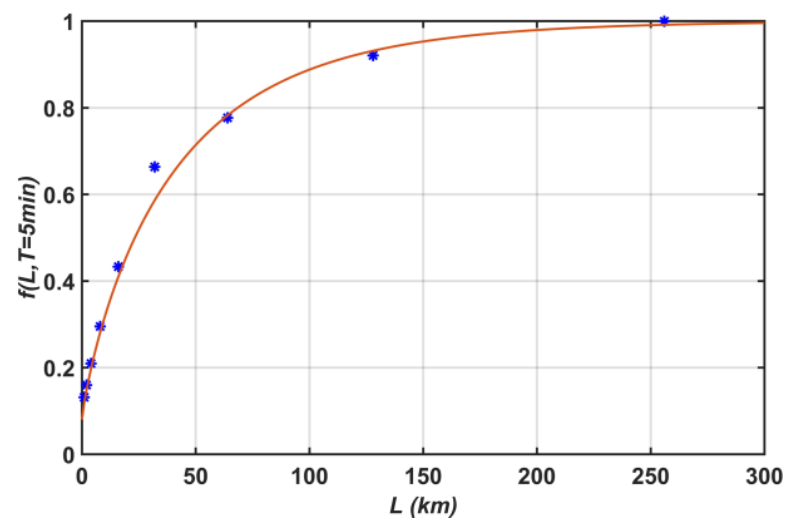

Fig. 9: Probability of rain occurrence for different spatial integration scales. The experimental points were obtained from UK NIMROD rain radar maps for Portsmouth in the southern UK. The best fit is based on the parameters $f=0.09, e=0.042$ and $g=0.85$.

It should be apparent that for small spatial integration scales $f(L \rightarrow 0, T=5 \mathrm{~min}) \rightarrow f$ and also, $f(L)$ monotonously increases up to $f(L \rightarrow \infty, T=5 \mathrm{~min}) \rightarrow 1$. Quite remarkably, the authors in [9] also found that rain intermittence in the time domain also obeys the same experimental formula:

$$
f(L=1 \mathrm{~km}, T)=1-(1-f) e^{-m T^{n}}
$$

The best fit with experimental rain radar data is exemplified in Fig. (10).

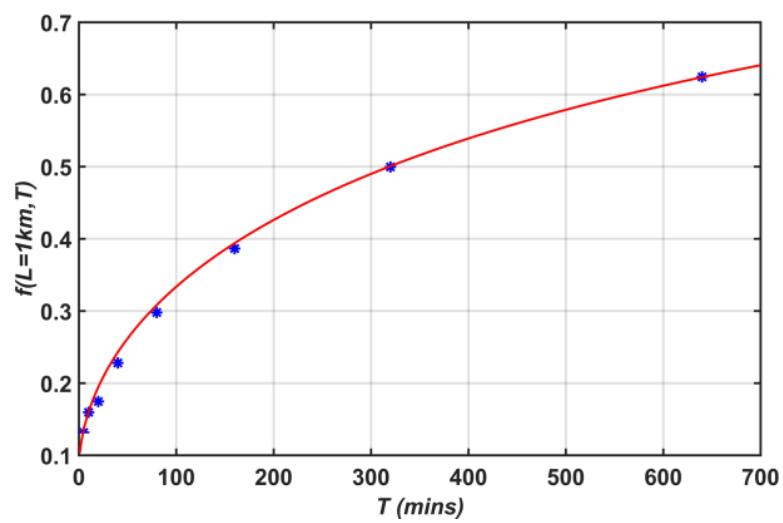

Fig. 10: Probability of rain occurrence for different temporal integration scales. The experimental points were obtained from UK NIMROD rain radar maps for Portsmouth in the southern UK. The best fit is based on the parameters $f=0.09, e=0.0256$ and $g=0.55$.

We note that independently and at about the same time as the authors in [9], Kundu and Siddani in [13] found the exactly the same empirical equations for the probability of rain occurrence based on raingauge data. They also interestingly try and provide a phenomenological Markov chain type model for explaining the form of Eq. (29) and Eq. (30).

In view of the above, it therefore seems adequate to combine these two equations to give the space-time prediction formula for the rain occurrence probability at all space and time averaging scales:

$$
f(L, T)=1-(1-f) e^{-e L^{g}} e^{-m T^{n}}
$$

and thus we obtain the space-time scaling formula for rain intermittence:

$$
\frac{f(L, T)}{f\left(L^{\prime}, T^{\prime}\right)}=\frac{1-(1-f) e^{-e L^{g}} e^{-m T^{n}}}{1-(1-f) e^{-e L^{\prime g}} e^{-m T^{\prime}}}
$$

We have thus demonstrated that (i) the mean of rainfall rate is invariant upon space-time integration and is equal to $\mu_{R}$, the mean of point rainfall rate. The variance of space-time integrated rainfall rate is $\sigma_{R}^{2}(L, T)=\gamma(L) \gamma(T) \sigma_{R}^{2}$ where $\sigma_{R}^{2}$ is the variance of point rainfall rate. The probability of rain occurrence is given by Eq. (31). This implies that the lognormal parameters of space-time integrated rainfall rate depend on the space and time scales of integration i.e. the pdf of space-time integrated rainfall rate is:

$$
f(R)=\left\{\begin{array}{c}
1-f(L, T) \\
\frac{f(L, T)}{\sqrt{2 \pi} \sigma R} \exp \left(-\frac{1}{2}\left(\frac{\ln R-m(L, T)}{\sigma(L, T)}\right)^{2}\right)
\end{array}\right.
$$

and the two lognormal parameters for the 'space-time' integrated pdf are: 


$$
\begin{gathered}
m(L, T)=\ln \left(\frac{\mu_{R}}{f(L, T)}\right)-\frac{1}{2}-\frac{f(L, T) \sigma_{R}^{2}(L, T)}{\mu_{R}} \\
\sigma(L, T)=\sqrt{1+\frac{f(L, T) \sigma_{R}^{2}(L, T)}{\mu_{R}}}
\end{gathered}
$$

We have plotted the complementary cumulative distribution of integrated rainfall rate for different integration periods assuming a fixed small spatial integration interval i.e. we have plotted:

$$
P_{r}\{R>x \mid x>0\}=f(L, T) \times Q\left(\frac{\ln R-m(L, T)}{\sigma(L, T)}\right)
$$

This is shown in Fig. (11) demonstrating the significance reduction in exceeded (extreme) rainfall rates for small percentages for increasing integration times. We also note that 1 second and 1 minute integration times yield very similar distribution function. A similar pattern (for brevity not shown in this paper) would be obtained when looking at the impact of the spatial integration interval. Larger spatial intervals reduce the probability of rain exceedence.

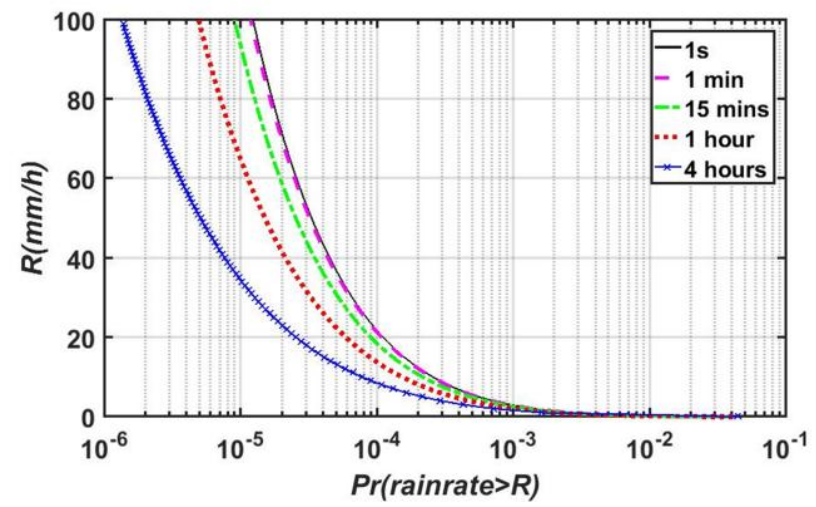

Fig. 11: Complementary cumulative distribution function of rainfall rate for different integration times $(L=100 m)$. This includes rain intermittence where we assume that $f=0.09$ for point rainfall rate.

\section{B. Fractional rainy area}

In this section, we consider a $2 \mathrm{D}$ flat map $\mathcal{M}$ with total geographical area $\mathcal{A}_{\mathcal{M}}$. At a particular instant in time typically representative of expectable meteorological conditions, there will be a population of rain cells covering a rainy area $\mathcal{A} \leq \mathcal{A}_{\mathcal{M}}$ scattered over $\mathcal{A}_{\mathcal{M}}$. Over many maps, we must have:

$$
\overline{\mathcal{A}}=f \mathcal{A}_{\mathcal{M}}
$$

where $\overline{\mathcal{A}}=E\{A \mid R>0\}$ denotes the average rainy area on $\mathcal{M}$. Let us denote the cdf of rainfall rate by $F(R)=$ $P_{r}\{$ Rainrate $<R \mid R>0\}=\frac{Q(\ln R-m)}{\sigma}$ which is the cdf conditioned on the fact that it is actually raining $R>0$. The $\operatorname{cdf} F(R)$ is thought of as the cdf of (above zero) rainfall rate over the map $\mathcal{M}$ which by our model definition is lognormal with parameters $(m, \sigma)$. Provided the total area $\mathcal{A}_{\mathcal{M}}$ is large enough, a typical map $\mathcal{M}$ will contain many rain storms or rain cells at different stages of their evolution and thus we can expect that the areal conditional pdf of rain over $\mathcal{M}$ will be identical to that at a point on $\mathcal{M}$ over a long time. In fact, we can expect that the conditional area $\overline{\mathcal{A}}(R>x) \leq \overline{\mathcal{A}}$ for which
$R>x$ on $\mathcal{M}$ must satisfy:

$$
\overline{\mathcal{A}}(R>x)=\overline{\mathcal{A}} \times \int_{x}^{\infty} d F(R)
$$

We note that when $x \rightarrow 0^{+}$, the integral is unity and thus the conditional rainy area for which $R>x$ tends towards the total rainy area $\overline{\mathcal{A}}$ as required. We now introduce the fractional area factor $f(R>x) \leq 1$ for which $R>x$ over the whole map $\mathcal{M}$ :

$$
\begin{aligned}
f(R>x) & =\frac{\overline{\mathcal{A}}(R>x)}{\mathcal{A}_{\mathcal{M}}} \\
& =f Q\left(\frac{\ln R-m}{\sigma}\right)
\end{aligned}
$$

This equation is a mere statement that the areas associated with larger rainfall rates tend to be smaller. This simple expression can furthermore be generalized to arbitrary spatial and temporal integration intervals by replacing the three lognormal parameters by $f(L, T), m(L, T)$ and $\sigma(L, T)$ that were described above.

We note that the analysis for the fractional rainy area has been verified by experiments. In particular, the volume of rain $V$ collected over a rainy area $\mathcal{A}$ over a duration $T$ is:

$$
V(R>0)=\int_{\mathcal{A}} \int_{T} R(\mathbf{x}, t) d \mathbf{x} d t=\overline{\mathcal{A}} \bar{R} T
$$

where we have used the first mean value theorem for evaluating the two integral. The quantity $[\overline{\mathcal{A}} T]$ is called the $\mathcal{A T I}$, the Area Time Integral. Eq. (41) indicates that the total volume of rain is proportional to the average rainfall rate $\bar{R}$ and the constant of proportionality is the $\mathcal{A T I}$. Such a relationship is described and experimentally verified by [19] and [20]. Using some of their information, we shall now demonstrate that this is equivalent to predicting Eq. (40). For this, we also introduce the average rainfall rate for which $R>x$ :

$$
\bar{R}(R>x)=\frac{\int_{x}^{\infty} R d F(R)}{\int_{x}^{\infty} d F(R)}
$$

The volume of collected rain for which the rainfall rate satisfies $R>x$ can be expressed by slightly modifying Eq. (41) giving $V(R>x)=\overline{\mathcal{A}}(R>x) \bar{R}(R>x) T$. After suitable substitution from previous equations, it is easy to verify that:

$$
V(R>x)=\overline{\mathcal{A}} T \times \int_{x}^{\infty} R d F(R)
$$

Requiring that the total volume of rain is conserved we can also write that $V(R>x)=\mathcal{A}_{\mathcal{M}} \mu_{R} T$ where $\mu_{R}$ is the map average rainfall rate. So:

$$
f(R>x)=\mu_{R} T \frac{\overline{\mathcal{A}}(R>X)}{V(R>0)}=\mu_{R} \frac{\int_{x}^{\infty} d F(R)}{\int_{x}^{\infty} \operatorname{RdF}(R)}
$$

The integral in the denominator is just the mean rainfall rate conditioned on actual rain occurring i.e. $\bar{R}=E[R \mid R>0]=$ $\exp \left(m+\frac{\sigma^{2}}{2}\right)$. Using Eq. (2), we see that Eq. (44) is the same as in Eq. (40).

\section{CONCLUSION}

In conclusion, the main objectives of this study can be 
summarized as: 1) investigation of rain/no rain condition for planning and simulation of HF radio wave communication systems; 2) characterization of the rainfall field within a wide range of space-time scales. With this in mind, we described a new comprehensive space-time model of point rainfall rate and its associated rainfield synthesizer called GRAM. Clearly, one of the main advantages of GRAM is that the rainfall rate process is differentiable. This is not, for example, the case in alternative models such as the Maseng-Bakken Model in [21].

We investigated the integrated rainfall rate fields through averaging the rainfall rate over an area of size $L \times L$ and/or over a period of time $T$. Remarkably, a good experimental fit has been achieved between the theoretical correlation function and the empirical radial correlation function $c_{R}^{\text {exp }}(r)$ of rainfall rate measured from rain radar networks using the proposed formula. This level of fit is self-evident and demonstrates that the exponential model can provide a strong argument for modeling the correlation function of rainfall rate in both space and time domains. In addition, we also looked at the impact of space and time averaging on rain intermittence and found that the dynamic characteristics of rainfall rate will become much slower with long range of spatial and temporal scale dependence. Furthermore, we have developed a new mathematical model that delivers good approximation of the probability of rain occurrence for different spatial and temporal integration scales. As such, this proposed model has the potential to provide significant cost reductions in satellite communication system deployment by better integrating the strengths of both radar systems and rain gauge networks. Finally, we provided the analysis of fractional rainy area in details so that the volume of rain has been well modelled. Using these studied quantities the rainfall rate field can be reasonably generated to simulate the instantaneous joint fade experienced by all the links in an arbitrary microwave network.

In addition, we reckon that the proposed equations for modeling the rainfield characteristics have some limitations: they are only valid for cases where either the spatial integration length or the temporal integration length is constant, whilst the other is changing. Since rain is highly variable in both space and time simultaneously, our future work will focus on characterizing the 3D space-time rainfall fields in 3D space-time domain follow the lead given in [11].

\section{ACKNOWLEDGMENT}

The authors would like to thank the British Atmospheric Data centre (BADC), which is part of the NERC National Centre for Atmospheric Science (NCAS), and the British MetOffice for providing access to the NIMROD rain radar data sets.

\section{APPENDIX: DESCRIPTION OF TURNING BAND METHOD}

The Turning Band Method (TBM) introduced in [22] is an elegant algorithm for generating $N$ - dimensional Gaussian fields on a Cartesian grid with arbitrary correlation function. In this paper, we are interested in the synthesis of 2D Gaussian field maps with zero mean, unit variance and isotropic correlation function $c_{g}(r)$ given for example by Eq. (19). The algorithm is described as follows.
1. Choose an arbitrary origin $\mathcal{O}$ in $\mathbb{R}^{2}$ and generate $L$ lines labeled by index $i$ passing through the origin with unit vector $\boldsymbol{u}_{i}$ with phase uniformly distributed over $[0,2 \pi)$.

2. Along each line $i$, generate a $1 \mathrm{D}$ discrete random process $\mathrm{z}_{i}(\zeta)$ with zero mean and covariance $c_{g}(\zeta)$ where $\zeta$ is the coordinate on line $i$. This is done by computing the spectral density function

$$
S_{1}(w)=\frac{1}{2} \int_{0}^{\infty} r c_{g}(r) J_{0}(w r) d r
$$

and then synthesizing the random time series $\mathrm{z}_{i}(\zeta)$ on line $i$ using sum over $M$ harmonics:

$$
\mathrm{z}_{i}(\zeta)=2 \sum_{k=1}^{M}\left[S_{1}\left(w_{k}\right) \Delta w\right]^{\frac{1}{2}} \cos \left(w_{k}^{\prime} \zeta+\emptyset_{k}\right)
$$

where $\emptyset_{k}$ are independent random phase over $[0,2 \pi), w_{k}=$ $(k-0.5) \Delta w$ and $w_{k}^{\prime}=w_{k}^{\prime}+\delta w$ for $k=1,2, \ldots, M$. The spectrum is truncated to $[-\Omega, \Omega]$ and so $\Delta w=\frac{\Omega}{M}$.

3. Onto line $i$, orthogonally project each point $\mathcal{P}$ with coordinates $\mathbf{x}$ of the Gaussian field where we want to generate values and assign to each point $\mathcal{P}$ the coordinate on line $i$. Assign to point, the value:

$$
g(\mathbf{x})=\frac{1}{\sqrt{L}} \sum_{i=1}^{L} \mathrm{z}_{i}\left(\mathbf{x}_{P} \cdot \boldsymbol{u}_{i}\right)
$$

In practice, the authors in [22] recommend that at least 20 lines $(L \gtrsim 20)$ should be used for good accuracy.

\section{REFERENCES}

[1]. R. K. Crane, "Electromagnetic wave propagation through rain", WileyInterscience, 1996.

[2]. G. Yang, "Rainfall Rate Modelling For European Satellite Networks", $\mathrm{PhD}$ thesis, University of Portsmouth, 2016.

[3]. B. Gremont. Et al, "Comparative analysis and performance of two predictive fade detection schemes for Ka-band fade countermeasures", IEEE Journal on selected areas in communications, Vol. 17 No2, 1999, pp 180-192.

[4]. B. C. Grémont and M. Filip, "Spatio-temporal rain attenuation model for application to fade mitigation techniques", Antennas and Propagation, IEEE Transactions on, 52(5), 2004, pp. 1245-1256.

[5]. T. L. Bell and P. K. Kundu, "A Stochastic Model of Space-Time Variability of Mesoscale Rainfall: Statistics of Spatial Averages", Water Resources Research, Vol. 39, 2003.

[6]. A. D. Panagopoulos and J. D. Kanellopoulos, "On the rain attenuation dynamics: spatial-temporal analysis of rainfall rate and fade duration statistics", International Journal of Satellite Communications and Networking, 21(6), 2003, pp. 595-611.

[7]. M. Valeria. Et al, "Study of the rainfall dependence structure using radar and rain gauge data", International workshop Advances in statistical Hydrology, Taormina, Italy, May 23-25 2010.

[8]. L. Luini and C. Capsoni, "The impact of space and time averaging on the spatial correlation of rain", Radio Science, 47(3), 2012.

[9]. G. Yang, B. Gremont, D. Ndzi and D. J. Brown, "Characterization of rain fields for UK satellite networks", $\mathrm{Ka}$ and Broadband Communications: navigation and Earth observation conference, 3-5 Oct 2011, Palermo, Italy.

[10]. T. L. Bell, "A Space-Time Stochastic Model of Rainfall for Satellite Remote-Sensing Studies", J. Geophysical Research, Vol. 92, No D8, pp. 9631-9643, August, 1987.

[11]. T. L. Bell and P. K. Kundu, "A study of the sampling error in satellite rainfall estimates using optimal averaging of data and a stochastic model", Journal of Climate, 9(6), 1996, pp. 50-57.

[12]. P. K. Kundu and T. L. Bell, "Space-time scaling behavior of rain statistics in a stochastic fractional diffusion model", Journal of Hydrology, 322, 2005, pp. 49-58.

[13]. P. K. Kundu and R. K. Siddani, "Scale dependence of spatiotemporal intermittence of rain", Water Resources Research, Vol. 47(8), W08522, Aug 2011. 
[14]. G. Yang Et al, "The Impact of Spatial-Temporal Averaging on the Dynamic-Statistical Properties of Rain Fields", has been submitted to IEEE for review.

[15]. P. K. Kundu and R. K. Siddani, "A New Class of Probability Distributions for Describing the Spatial Statistics of Area-averaged Rainfall”, Journal Geography Research, 112, 2007, D18113.

[16]. B. Kedem and L. S. Chiu, "On the lognormality of rain rate", Vol. 84(4), pp. 901-905, February 1, 1987.

[17]. A. Papoulis and S. U. Pillai, "Probability, Random Variables and Stochastic Proccesses", $4^{\text {th }}$ Edition, McGraw Hill, 2002.

[18]. G. Christakos, "Random Field Models in Earth Sciences", Dover Publications, 1992.

[19]. E. A. Eltahir and R. L. Bras, "Estimation of the fractional coverage of rainfall in climate models", Journal of Climate, Vol. 6, pp. 639-644, April 1993.

[20]. D. Atlas, D. Rosenfeld and D. A. Short, "The estimation of convective rainfall by area integrals. 1. The theoretical and empirical basis", J. Geophysical Research, Vol. 95, No D3, pp. 2153-2160, 1990.

[21]. T. Maseng and P. M. Bakken, "A stochastic dynamic model of rain attenuation", IEEE Trans. Communications, Volume: 29, Issue: 5, May 1981, Pages: 660-669.

[22]. A. Mantoglou and J. L. Wilson, "The turning band method for simulation of random fields using line generation by a spectral method", Water Resources Research, Vol. 18, No 5, Oct 1982, pp. 1379-1394.

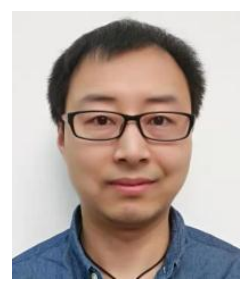

Guangguang Yang was born in China on July 17, 1986. He received the diploma (Distinction) from the Xinxiang College, Xinxiang, China, in 2008 and the Bachelor's degree (First Class Honors) and the Ph.D. degree in electronic engineering from University of Portsmouth, Portsmouth, UK, in 2010 and 2016, respectively. He is now a senior technical consultant at infosystech Ltd, London, UK. His current research interests include earth-space, terrestrial and wideband propagation, and satellite communications.

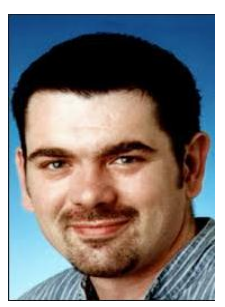

Boris Christian Gremont (Jan 9, $1969 \sim$ Dec 20, 2013) received the DUT from the Universitary Institute of Technology (IUT), Grenoble, France, in 1992 and the Bachelor's degree (First Class Honors) in engineering and the Ph.D. degree in electrical electronic engineering, Coventry, U.K., in 1994 and 1998, respectively. After postdoctoral research at the Microwave Telecommunication Systems Research Group (MTSRG), University of Portsmouth, Portsmouth, U.K., he was appointed Senior Lecturer in the Department of Electronic \& Computer Engineering. He was also involved in the European COST 280 action "Propagation Impairment Mitigation for Millimeter Wave Radio Systems" and he also contributed to the UK's RAINMAP project (Fractal Rain Modelling for Millimeter Wave Propagation).

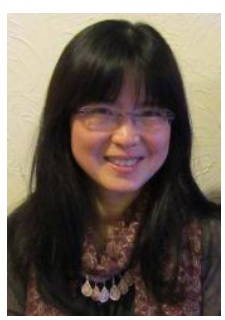

Linda Yang received her $\mathrm{PhD}$ degree in computer science from Peking University, China in 1999, and her MS and BE degrees in computer software in 1996 and 1993, respectively. She is now a senior lecturer at the University of Portsmouth, UK. Prior to that, she was a lecturer at the Robert Gordon University, a senior research fellow at the University of St Andrews and Cardiff University, UK. Her research interests include recommendation system, information retrieval, and data mining as well as their applications in social networks, health-informatics and business. She has worked on UK and European funded research projects and published over 50 papers in refereed journals and conferences. She is a member of the IET, WES and HEA.

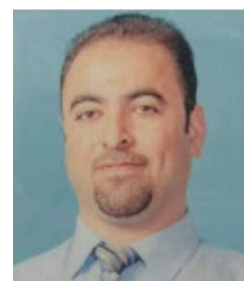

Mohammed E. Ibrahim received his B.S in Software Engineering from College of Technology, Irap in 2005. He received his M.S. in Computer Network Administration and Management from University of Portsmouth, United Kingdom in 2009. He is currently a PhD student in the school of engineering, University of Portsmouth, UK. His research interests are in the areas of artificial intelligence, Data Integration, Recommendation Systems, Semantic web, Ontology Engineering, Data Mining.

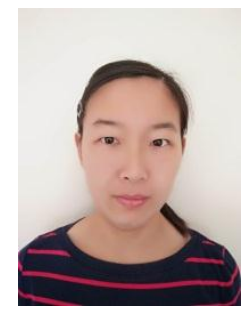

Li Bai was born in China on July 24, 1986. She received the diploma (Distinction) from the Xinxiang College, Xinxiang, China, in 2008 and the Bachelor's degree from the Henan Polytecnic University in 2010, respectively. She worked as a physics teacher in China between 2011 and 2015 and later received her MSc degree in electronic engineering from University of Portsmouth, Portsmouth, UK, in 2017. 